\title{
Antibiotiques pour la prophylaxie des infections hématogènes tardives de prothèses articulaires
}

\author{
M. Rossi', W. Zimmerlib , H. Furrer', G. Zanetti ${ }^{d}$, K. Mühlemannc, M. G. Täuber ${ }^{c}$ pour la Société Suisse d'Infectiologie
}

Les patients porteurs de prothèses articulaires reçoivent fréquemment des antibiotiques avant des interventions invasives ou des traitements dentaires, par analogie avec la prophylaxie de l'endocardite bactérienne. Cette analogie n'est cependant pas fondée: en effet, les infections de prothèses articulaires ont une pathogenèse différente de celle de l'endocardite, et ne sont pas causées par les mêmes germes. De plus, l'efficacité d'une antibioprophylaxie chez les porteurs de prothèses n'a jamais été démontrée scientifiquement. Pour cette raison, il n'y a pas d'indication généralisée à une telle prophylaxie. En revanche, il convient de rechercher activement toute infection touchant une autre partie du corps, et de la traiter rapidement. Une antibioprophylaxie peut exceptionnellement être appropriée pour des interventions pratiquées chez certains patients qui courent un risque accru d'infection hématogène de prothèse en cas de bactériémie. Quant aux opérations pratiquées de routine sous antibioprophylaxie, le choix du régime prophylactique est inchangé pour des patients porteurs de prothèses articulaires.

\footnotetext{
a Kantonsspital Luzern

b Medizinische Universitätsklinik, Kantonsspital Liestal

c Klinik und Poliklinik für Infektiologie, Universitätsspital Insel, Bern

d Service des maladies infectieuses,

Université de Lausanne
}

Correspondance:

Dr Marco Rossi

Hôpital cantonal

Infectiologie

CH-6000 Lucerne 16

Tél. 0412051111

E-mail: marco.rossi@ksl.ch

\section{Introduction}

Les médecins de premiers recours, les orthopédistes et les dentistes cherchent souvent à s'informer sur la nécessité d'une antibioprophylaxie chez les patients porteurs de prothèses articulaires lors de procédure invasive, par analogie à la prophylaxie de l'endocardite bactérienne. Ceci laisse présumer que la prophylaxie est souvent utilisée dans ces situations. Nous avons dès lors cherché à répertorier les arguments existants pour ou contre une antibioprophylaxie chez les patients porteurs de prothèses articulaires, et tenté d'en déduire des recommandations pratiques.

\section{Méthode}

La littérature traitant de l'antibioprophylaxie chez des porteurs de prothèses articulaires a été identifiée au moyen d'une recherche dans Medline, ainsi que par la revue de chapitres de livres spécifiques et autres publications relevantes. L'examen systématique des données disponibles nous a fait conclure que nous ne disposions pas d'arguments suffisant à l'élaboration de directives officielles («Guidelines») en raison de l'absence d'études contrôlées [1]. Pour cette raison, nous avons décidé de concevoir cet article comme un ensemble de «recommandations», dans le but d'aider le praticien à prendre une décision pour ou contre une antibioprophylaxie en tenant compte des arguments disponibles [2]. Nous avons soumis le manuscrit pour vérification aux services d'infectiologie des cliniques universitaires de Suisse.

\section{Infections chez les porteurs de prothèses articulaires}

La mise en place d'une prothèse articulaire apporte une nette amélioration de la qualité de vie à de nombreux patients. Bien que rare, la surinfection d'une telle prothèse constitue la complication la plus sévère de ces interventions. Selon leur moment d'apparition, on distingue des infections précoces (survenant par définition dans les 3 mois qui suivent l'implantation), des infections différées (entre 3 mois et 2 ans) et des infections tardives (tab. 1). Alors que les infections précoces découlent essentiellement d'une contamination exogène au cours de l'intervention (principalement à partir de la flore cutanée du patient), les infections plus tardives sont dues à un ensemencement hématogène de la prothèse [3]. Quant aux infections différées, elles sont également péri-opératoires, mais c'est la manifestation clinique qui en est différée de par la faible virulence des pathogènes en cause (staphylocoques à coagulase négative, Corynebacterium spp, etc.).

En raison de la morbidité considérable de ces infections, de leur létalité et des coûts élevés qu'elles engendrent, la question de leur prophylaxie est importante. Alors que l'antibioprophylaxie s'est largement répandue pour la prévention des infections précoces [4], cette pratique est discutable en ce qui concerne les infections tardives lors de situations à risque.

Ainsi, dans une étude, l'indication à une antibioprophylaxie avant une intervention dentaire chez un patient porteur de prothèse 
Tableau 1

Pathogenèse et bactéries causales en fonction du temps de survenue de l'infection.

\begin{tabular}{llll} 
& Infection précoce & Infection différée & Infection tardive \\
\hline Délai depuis I'opération & $<3$ mois & 3 mois-2 ans & $>2$ ans \\
\hline Pathogenèse probable & $\begin{array}{l}\text { Inoculation } \\
\text { péri-opératoire }\end{array}$ & $\begin{array}{l}\text { Inoculation } \\
\text { péri-opératoire }\end{array}$ & $\begin{array}{l}\text { Dissémination } \\
\text { hématogène }\end{array}$ \\
& Flore cutanée: & Flore cutanée: & Divers \\
Pathogènes en cause & S. aureus & - Staphylocoques & (le plus souvent \\
& & à coagulase négative & S. aureus) \\
& & - Corynebacterium spp & \\
& & - Propionibacterium spp &
\end{tabular}

de hanche était-elle posée de façon très différente par un chirurgien orthopédique (indication retenue dans $77 \%$ des cas) ou par un chirurgien maxillaire (retenue dans $29 \%$ des cas) [5]. En l'absence de données cliniques, la plupart des patients seraient traités par analogie avec la prophylaxie de l'endocardite bactérienne [6]. Plusieurs arguments s'opposent pourtant à cette analogie: les différences anatomiques, la vascularisation, le spectre des pathogènes potentiels, ou encore le mécanisme et l'incidence des infections de prothèses. Nous avons répertorié ci-dessous les arguments pour ou contre une antibioprophylaxie, et limité notre revue à la prévention des infections tardives hématogènes, aussi bien chez des patients immuno-compétents qu'immuno-compromis.

\section{Considérations sur la pathogenèse}

La colonisation hématogène sélective expérimentale d'implants extravasculaires a été décrite en 1985 déjà [7]. Le risque d'une telle colonisation culmine peu de temps après l'implantation de la prothèse. Il est conditionné, entre autres, par les sources d'infections que sont l'hématome opératoire, les cathéters intra-veineux, les rétentions urinaires post-opératoires et les infections des voies urinaires [3]. Le risque infectieux lié à des procédures invasives, dentaires ou autres, semble minime, et son lien causal ne repose dans ces situations que sur des descriptions de cas ou des modèles animaux [8]. Le type de pathogène impliqué dans les infections hématogènes de prothèses parle contre un risque élevé lié aux interventions invasives aux niveaux dentaire ou maxillaire, de même que la faible intensité des bactériémies durant la plupart des opérations et les résultats de grandes études de cohorte. Les streptocoques viridans sont prédominants dans la flore orale, sont responsables de bactériémies induites par des traitements dentaires et sont les agents les plus importants d'endocardite bactérienne subaiguë [9]. En revanche ces streptocoques viridans ne causent que $2 \%$ des infections de prothèses [10]. Les germes les plus souvent en cause dans ces cas sont plutôt des staphylocoques (en particulier Staphylococcus aureus avec $38 \%$ ), alors que ces derniers ne représentent que le $0,005 \%$ de la flore orale et ne sont que peu retrouvés dans les bactériémies d'origine dentaire. De surcroît, les bactériémies provoquées par des interventions dentaires ou autres sont brèves et de faibles intensités [10, 11], alors que la colonisation hématogène d'un implant requière une bactériémie soutenue et de grandes quantités de bactéries ( $\geq 100$ colonies de $S$. aureus par ml de sang) [5].

$\mathrm{Au}$ cours d'une étude prospective, 14 sur 1278 porteurs de $S$. aureus au niveau nasal ont développé une bactériémie. La typisation a montré que la souche retrouvée dans les hémocultures était la même qu'au niveau nasal chez 12 de ces 14 porteurs (86\%) [12]. On peut en conclure qu'une importante proportion des bactériémies à $S$. aureus sont d'origine endogène. Ces données ne suffisent pas à justifier une stratégie préventive qui consisterait en un dépistage suivi le cas échéant d'une décolonisation [13].

\section{Données épidémiologiques}

Une étude de cohorte prospective [14] portant sur 6 ans a démontré une incidence d'infection de prothèse de $3 / 1000$ patients (correspondant à 50/100 000 personnes-années). Par comparaison, le taux d'incidence est de 1,7-3,8/100 000 personnes-années pour l'endocardite sur valve native [15] et de 40-400/100000 personnesannées pour l'endocardite sur valve prothétique [16]. Les infections cutanées semblent plus fréquemment à l'origine d'infections de prothèse que des interventions invasives. Elles ont également été identifiées comme un facteur de risque pour une arthrite septique au cours d'une étude prospective, avec un odds ratio de 27,2 (intervalle de confiance $95 \%: 7,6-97,1)$ [17].

Un résumé de 67 cas documentés d'infection hématogène tardive de prothèse articulaire (survenant plus d'une année après l'implantation) a révélé les foyers primaires suivants: 
Tableau 2

Fréquence de foyers primaires lors d'infections de prothèse par voie hématogène [31].

\begin{tabular}{ll} 
Foyer primaire probable & Fréquence relative (\%) \\
\hline Peau et tissus mous & 46 \\
\hline Dentition & 15 \\
\hline Voies urinaires & 13 \\
\hline Voies respiratoires & 16
\end{tabular}

Une étude prospective portant sur 80 patients avec une bactériémie documentée à $S$. aureus a révélé une infection hématogène de 15/44 (34\%) prothèses articulaires et de 1/15 (7\%) autres implants orthopédiques [18].

La bactériémie à $S$. aureus n'avait pas de foyer d'origine connu dans $25 \%$ des infections d'implants; elle découlait dans 31\% des cas d'une infection de la peau ou des tissus mous, dans $25 \%$ d'un autre foyer musculo-squelettique, et dans 19\% d'une infection de cathéter intravasculaire. Les prothèses de genou tendaient à être plus touchées que les prothèses de hanche (50\% vs $26 \%$, ns).

La Porte a publié une analyse rétrospective de 2973 prothèses de hanche. Parmi 52 infections tardives, 3 (6\%) étaient associées à des interventions dentaires. Deux de ces trois patients infectés souffraient de maladies sous-jacentes (diabète, arthrite rhumatoïde), tous avaient subi une intervention d'une durée supérieure à 45 min. et aucun n'avait reçu d'antibioprophylaxie [19].

\section{Facteurs prédisposants}

Les conditions suivantes ont été identifiées comme des facteurs prédisposant à une infection de prothèse articulaire [20]: polyarthrite rhumatoïde (risque relatif; 1,8 au niveau de la hanche, 2,4 au niveau du genou), hémophilie, diabète sucré, traitement par stéroïde ou méthotrexate, immunosuppression maladive ou médicamenteuse. Aucune prédisposition aux infections tardives n'a été documentée chez les patients souffrant d'infection par le VIH.

Dans une étude cas-témoin portant sur 462 infections, l'analyse multivariée révélait les facteurs de risque suivants [21]: la présence d'une infection superficielle de plaie (OR 35,9, CI $95 \%$, 8,3-154,6), un score de risque chirurgical (basé sur la durée de l'intervention, le risque anesthésiologique et la classe de contamination [22]) de 1 (OR 1,7, CI 1,2-2,3) ou 2 (OR 3,9, CI $2,0-7,5)$, ainsi qu'une néoplasie maligne (OR 3,1, CI 1,3-7,2). La polyarthrite rhumatoïde, diabète sucré et l'hémophile ne représentaient pas les facteurs de risque indépendants.

\section{Données sur I'efficacité de la prophylaxie}

Jusqu'ici, l'efficacité d'une antibioprophylaxie n'a pu être démontrée par des études cliniques. Des essais d'analyse risque-bénéfice parlent contre l'application généralisée de cette antibioprophylaxie: en se basant sur une incidence estimée de 30 infections hématogènes chez 100000 patients $(0,03 \%)$, l'étude de Norton prédit 40 réactions anaphylactiques, dont 4 létales, liées à l'administration prophylactique d'antibiotiques [23]. Des analyses coût-bénéfice ont été établies sur la base de modèles d'analyse décisionnelle: Jacobson [24] a ainsi calculé qu'une prophylaxie par céphalosporine orale entraînerait des dépenses de US\$ 500 000.- pour une année de vie gagnée, et de US\$ 480000 .pour une infection de prothèse évitée. Une autre analyse coût-bénéfice a estimé que l'administration prophylactique d'érythromycine serait liée à un rapport coût-efficacité marginal de US\$ 12 900.- par année de vie gagnée [25].

Tableau 3

Mesures visant à réduire les sources potentielles de bactériémies.

\begin{tabular}{lll} 
Problème clinique & Exemples & Mesures \\
$\begin{array}{l}\text { Foyer infectieux } \\
\text { (avant l'intervention prévue) }\end{array}$ & $\begin{array}{l}\text { Avant chirurgie maxillaire: carie dentaire, } \\
\text { parodontose; avant une cystoscopie: } \\
\text { infection des voies urinaires }\end{array}$ & $\begin{array}{l}\text { Traitement de l'infection } \\
\text { avant l'intervention }\end{array}$ \\
\hline Infection & $\begin{array}{l}\text { Infection cutanée, ulcère de décubitus, } \\
\text { infection des voies urinaires, parodontose }\end{array}$ & $\begin{array}{l}\text { Investigation et traitement } \\
\text { dirigé }\end{array}$ \\
\hline Corps étranger & Cathéter vésical, cathéter intravasculaire & Ablation dès que possible \\
\hline $\begin{array}{l}\text { Intervention élective avec risque } \\
\text { accru de bactériémie }\end{array}$ & $\begin{array}{l}\text { Résection trans-urétrale de la prostate, } \\
\text { tonsillectomie }\end{array}$ & $\begin{array}{l}\text { Ajournement à }>1 \text { an après } \\
\text { l'implantation de la prothèse }\end{array}$
\end{tabular}


Tableau 4

Patients à risque accru d'infection de prothèse par voie hématogène [27, 32].

Implantation d'une prothèse articulaire au cours des 12 mois précédents

- Polyarthrite rhumatoïde sous immunosuppression

- Polyarthrite rhumatoïde avec risque additionnel (diabète sucré, changement de prothèse)

- Hémophilie

\section{Tableau 5}

Interventions liées à un risque accru de bactériémie [27, 32]

- Intervention dentaire d'une durée $>45$ minutes

- Intervention dentaire sur une gencive sévèrement altérée

- Intervention en urgence sur un site présomptivement infecté (peau, voies urinaires, autres)

\section{Tableau 6}

Choix de l'antibiotique pour la prophylaxie.

\begin{tabular}{|c|c|c|}
\hline & Premier choix & En cas d'allergie à la pénicilline \\
\hline 1 heure avant l'intervention & $\begin{array}{l}\left.\text { Amoxi/Clav (Augmentin }{ }^{\circledR}\right) \\
2 \text { g p.o. }\end{array}$ & $\begin{array}{l}\text { Clindamycin (Dalacin- }{ }^{\circledR} \text { ) } \\
600 \text { mg p.o. }\end{array}$ \\
\hline 4 heures après l'intervention & $\begin{array}{l}\text { Amoxi/Clav (Augmentin }{ }^{\otimes} \text { ) } \\
1 \text { g p.o. }\end{array}$ & $\begin{array}{l}\text { Clindamycin (Dalacin-C }{ }^{\circledR} \text { ) } \\
600 \text { mg p.o. }\end{array}$ \\
\hline
\end{tabular}

\section{Recommandations}

Puisqu'il n'est pas possible de fonder la prophylaxie des infections de prothèses articulaires sur celle de l'endocardite, ni quant à ses indications, ni quant au choix des antibiotiques, il faut s'efforcer d'établir des directives simples et praticables sur la base des rares résultats d'études, partiellement contradictoires, et sur des considérations théoriques [26, 27]. C'est dans ce contexte que nous formulons ici 4 recommandations spécifiques.

\section{Réduction du risque de bactériémie}

Etant donné que la grande majorité des infections tardives font suite à des bactériémies, la reconnaissance, le traitement et la prévention des circonstances cliniques favorisant la survenue de bactériémies constituent la stratégie la plus importante pour la prévention de ces infections. Il faut ajouter à cela le rôle clé d'une évaluation médicale attentive et compétente des patients porteurs de prothèses articulaires. Le tableau 3 fournit des exemples de réductions possible du risque de bactériémie [28]. Une hygiène dentaire soigneuse assurée par le patient lui-même en fait partie [29]. En cas d'infection cutanée floride et abcédante, il convient d'associer une antibiothérapie au traitement chirurgical (incision, respectivement drainage).

\section{Pas d'antibioprophylaxie généralisée}

Au delà d'une année après l'implantation d'une prothèse articulaire, l'administration de routine d'antibiotique n'est pas indiquée chez le patient immunocompétent avant une intervention dentaire (en l'absence d'infection floride), une cystoscopie (en l'absence d'une infection des voies urinaires), étant donné que le risque d'infection est minime et que nous ne disposons pas d'argument suggérant qu'une indication large pourrait avoir un rapport risque-bénéfice favorable.

\section{Situations exceptionnelles avec risque infectieux accru}

Même chez les patients particulièrement susceptibles (tab. 4) et lors d'interventions associées à un risque très élevé de bactériémie (tab. 5), il n'y a pas d'indication généralisée à une antibioprophylaxie. Si l'on opte de cas en cas pour une prophylaxie chez des patients à risque qui subissent des interventions à risque, le choix de l'antibiotique devrait être fondé sur les pathogènes les plus probablement responsables d'une infection hématogène de prothèse (principalement des staphylocoques, plus rarement des streptocoques). Ceci conduirait par exemple à choisir l'amoxicilline/clavulanate (Augmen$\left.\operatorname{tin}^{\circledR}\right)$, avec pour alternative la clindamycine (Dalacin ${ }^{\circledast} \mathrm{C}$ ) ou une céphalosporine de première génération, la céfazoline $\left(\mathrm{Kefzol}^{\circledR}\right)$.

\section{Antibiotiques péri-opératoires}

En cas d'intervention invasive de toute sorte pour laquelle une antibioprophylaxie péri-opératoire est indiquée chez tout patient, le régime prophylactique établi est également valable pour un patient porteur d'une prothèse articulaire.

Sur des bases analogues, un «scientific statement» de l'American Heart Association [30] s'est clairement prononcé contre une antibioprophylaxie généralisée chez les porteurs d'implants autre que des valves cardiaques. La recommandation vaut aussi bien pour les patients immunocompromis que pour les immunocompétents.

\section{Résumé}

1. Travaux d'assainissement dentaire avant l'implantation d'une prothèse. Maintient d'une bonne hygiène dentaire par le patient.

2. Pas d'antibioprophylaxie de routine chez les patients porteurs de prothèse articulaire lors d'interventions invasives. 
3. Antibiothérapie rapide des infections qui peuvent causer des bactériémies compromettant des implants (cf. tab. 2).

4. Recherche et prise en charge des conditions cliniques favorisant une bactériémie chez tous les patients porteurs de prothèse articulaire (cf. tab. 3).

5. Lors d'opération habituellement pratiquée sous antibioprophylaxie péri-opératoire, le même schéma prophylactique doit être appliqué au patient porteur de prothèse articulaire.

\section{Références}

1 Kish MA. Guide to development of practice guidelines. Clin Infect Dis 2001;32:851-4.

2 Obrist R, Zenger C. Legitimation und Wirkung von Standards am Beispiel von Guidelines. Schweiz Ärztezeitung 2004;85(5):231-5.

3 Hanssen AD, Osmon DR, Nelson CL. Prevention of deep periprosthetic joint infection. J Bone Joint Surg (Am) 1996;78(3):458-71.

4 Norden CW. Antibiotic prophylaxis in orthopedic surgery. Rev Infect Dis 1991;13(Suppl 10): S842-S846.

5 Sandhu SS, Reuben SF, Lowry JC, Morton ME. Who decides on the need for antibiotic prophylaxis in patients with major arthroplasties requiring dental treatment: is it a joint reponsibility? Am R Coll Surg Engl 1997;79:143-7.

6 Dajani AS, Taubert KA, Wilson W, Bolger AF, Bayer A, Ferrieri P, et al. Prevention of bacterial endocarditis. JAMA 1997;277(22):1794-801.

7 Zimmerli W, Zak O, Vosbeck K. Experimental hematogenous tnfection of subcutaneously implanted foreign bodies. Scand J Infect Dis 1985;17:303-10.

8 Blomgren G, Lindgren U. The susceptibility of total joint replacement to hematogenous infection in the early postoperative period: an experimental study in the rabbit. Clin Orthop 1980;151:308-12.

9 Watanakunakorn C, Burkert T. Infective endocarditis in large community teaching hospital, 1980-1990. Medicine 1993;72(2):90-102.

10 Wahl MJ. Myths of dental-induced prosthetic joint infection. Clin Infect Dis 1995;20:1420-5.

11 Little JW. Patients with prosthetic joints: are they at risk when receiving invasive dental procedures? Spec Care Dentist 1997;17(5):153-60.

12 von Eiff C, Becker K, Machka K, Stammer H, Peters G. Nasal carriage as a source of staphylococcal bacteremia. N Engl J Med 2001;344:11-6.

13 Archer GL, Climo MW. Staphylococcus aureus bacteremia - consider the source. N Engl J Med 2001;344:55-6.

14 Ainscow DAP, Denham RA. The risk of haematogenous infection in total joint replacements. J Bone Joint Surg (Am) 1984;66(4):580-2.
15 Bayer AS, Scheld WM. Endocarditis and Intravascular Infections. In: Mandell GL, Bennett JE, Dolin R (eds.). Principles and Practice of Infectious Diseases. $5^{\text {th }}$ ed. Philadelphia: Churchill Livingstone; 2000. p. 857-902.

16 Karchmer AW. Infections of Prosthetic Heart Valves. In: Waldvogel FA, Bisno AL (eds.). Infections Associated with Indwelling Medical Devices. $3^{\text {rd }}$ ed. Washington D.C.: ASM Press; 2000. p. $145-72$.

17 Kaandorp CJE, van Schaardenburg D, Krijnen P, Habbema DJF, van de Laar MAFJ. Risk factors for septic arthritis in patients with joint disease. Arthritis and Rheumatism 1995;38(12):1819-25.

18 Murdoch DR, Roberts SA, Fowler Jr. VG, Shah MA, Taylor SL, Morris AJ, et al. Infection of orthopedic prostheses after staphylococcus aureus bacteremia. Clin Infect Dis 2001;32:647-9.

19 La Porte DM, Waldman BJ, Mont MA, Hungerford DS. Infections associated with dental procedures in total hip arthroplasty. J Bone Joint Surg (Br) 1999;81-B:56-9.

20 Deacon BA, Pagliaro AJ, Zelicof SB, Horowitz HW. Prophylactic use of antibiotics for procedures after total joint replacement. J Bone Joint Surg (Am) 1996;78(11):1755-70.

21 Berbari EF, Hanssen AD, Duffy MC, Steckelberg JM, Ilstrup DM, Harmsen WS, et al. Risk factors for prosthetic joint infection: case-control study. Clin Infect Dis 1998;27:1247-54.

22 Surveillance. NNI. NNIS Report. Am J Infect Control 1996;24:380-8.

23 Norden CW. Prevention of bone and joint infections. Am J Med 1985;78(6B):229-32.

24 Jacobson JJ, Schweitzer S, DePorter DJ, Lee JJ. Antibiotic prophylaxis for dental patients with joint prostheses? A decision analysis. Int J Technol Assess Health Care 1990;6(4):569-87.

25 Tsevat J, Durant-Zaleski I, Pauker SG. Costeffectiveness of antibiotic prophylaxis for dental procedures in patients with artificial joints. Am J Public Health 1989;79(6):739-43.

26 ADA, AAOS. Antibiotic prophylaxis for dental patients with total joint replacements. American Dental Association, American Academy of Orthopedic Surgeons. J Am Dent Assoc 1997;128(7):1004-8.

27 Segreti J. Is antibiotic prophylaxis necessary for preventing prostitic device infection? Infect Dis Clin North Am 1999;13(4):871-7.

28 Osmon DR. Antimicrobial prophylaxis in adults. Mayo Clin Proc 2000;75:98-109.

29 Seymour RA, Whitworth JM. Antibiotic prophylaxis for endocarditis, prosthetic joints, and surgery. Dent Clin N Am 2002;46:635-51.

30 Baddour LM, Bettmann MA, Bolger AF, Epstein AE. Nonvalvular cardiovascular device-related infections. Circulation 2003;108:2015-31.

31 Maderazo EG, Judson S, Pasternak H. Late infections of total joint prostheses. Clin Orthop 1988;229:131-42.

32 Curry S, Philipps H. Joint Arthroplasty, Dental Treatment, and Antibiotics. A Review. J Arthroplasty $2002 ; 17(1): 111-3$. 